\title{
Effectiveness and toxicity of first-line methotrexate chemotherapy in low-risk postmolar gestational trophoblastic neoplasia: The New England Trophoblastic Disease Center experience
}

\author{
Izildinha Maestá a,e,f,*, Roni Nitecki ${ }^{\mathrm{b}}$, Neil S. Horowitz ${ }^{\mathrm{c}, \mathrm{d}, \mathrm{g}, \mathrm{h}}$, Donald P. Goldstein ${ }^{\mathrm{c}, \mathrm{d}, \mathrm{g}, \mathrm{h}}$, \\ Marjory de Freitas Segalla Moreira ${ }^{\text {a,e,f, }}$, Kevin M. Elias ${ }^{\text {c,d,g,h }}$, Ross S. Berkowitz ${ }^{\text {c,d,g,h }}$ \\ a Department of Gynecology and Obstetrics, Botucatu Medical School, UNESP-Sao Paulo State University, Botucatu, SP, Brazil \\ b Department of Obstetrics and Gynecology, Brigham and Women's Hospital Boston, MA, USA \\ ' Department of Obstetrics and Gynecology, Division of Gynecologic Oncology, Brigham and Women's Hospital, Boston, MA, USA

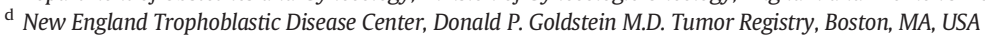 \\ e Trophoblastic Diseases Center of the Botucatu Medical School, UNESP-Sao Paulo State University, Botucatu, SP, Brazil \\ f Postgraduate Program of Gynecology, Obstetrics and Mastology of Botucatu Medical School, UNESP-São Paulo State University, Botucatu, SP, Brazil. \\ ${ }^{g}$ Dana Farber Cancer Institute/Harvard Cancer Center, Boston, MA, USA \\ ${ }^{\text {h }}$ Harvard Medical School, Boston, MA, USA
}

\section{H I G H L I G H T S}

- The study provides the most complete toxicity review of methotrexate in low-risk GTN.

- 8-day MTX-FA caused more toxicity but most toxicities were mild.

- 8-day MTX-FA had a higher remission rate than MTX infusion.

\section{A R T I C L E I N F O}

\section{Article history:}

Received 21 August 2017

Received in revised form 23 October 2017

Accepted 24 October 2017

Available online 29 October 2017

\section{Keywords:}

First-line methotrexate chemotherapy

Effectiveness

Toxicity

Low-risk gestational trophoblastic neoplasia

\begin{abstract}
A B S T R A C T
Objectives. To assess the outcomes and toxicity of first-line methotrexate (MTX) chemotherapy in low-risk postmolar gestational trophoblastic neoplasia (GTN) patients receiving 8-day methotrexate or one-day methotrexate infusion regimens.

Methods. This retrospective cohort study was conducted at the New England Trophoblastic Disease Center (NETDC), between 1974 and 2014, and included 325 patients with FIGO-defined low-risk postmolar GTN receiving first-line 8-day MTX/folinic acid (FA) or one-day MTX infusion and FA. Demographics, disease presentation, initial treatment plan, treatment outcome, and treatment-related adverse events were assessed.

Results. Sustained remission ( $84 \%$ vs $62 \%, p<0.001$ ) and need to switch to second-line therapy due to treatment-related adverse events ( $5.3 \%$ vs $0 \%, p=0.001$ ) were higher for 8-day MTX/FA compared to one-day MTX infusion. MTX resistance, however, was more frequent with one-day MTX (34.5\%) than with 8-day MTX/ FA $(7.3 \%, p<0.001)$. Relapse rates were similar with both regimens (3.0\%). Compared to one-day MTX infusion, 8-day MTX/FA was associated with significantly higher gastrointestinal disorders (48\% vs 24\%), abnormal laboratory findings ( $48 \%$ vs $28 \%$ ), eye disorders ( $37 \%$ vs $19 \%$ ) and general disorders ( $22 \%$ vs $5 \%)(p<0.001)$. Only infection frequency did not differ between 8-day MTX/FA and one-day MTX infusion (20\% vs 12\%, $p=0.083$ ).

Conclusions. This is one of the largest studies to comprehensively catalogue toxicities associated with 8-day MTX/FA and one-day MTX infusion. Although treatment-related adverse events were more frequent with 8day MTX/FA, these were all self-limited and resolved with no long-term sequelae. Given this and its higher effectiveness, 8-day MTX/FA remains the treatment of choice at NETDC for patients with low-risk postmolar GTN.
\end{abstract}

(C) 2017 Published by Elsevier Inc.

\footnotetext{
* Corresponding author at: Department of GYNOB, Botucatu Medical School, UNESP Sao Paulo State University, Av. Prof. Montenegro s/n, Botucatu, SP 18.618-687, Brazil.

E-mail address: imaesta@fmb.unesp.br (I. Maestá).
}

\section{Introduction}

Patients with gestational trophoblastic neoplasia (GTN) are classified into two prognostic groups on the basis of the extent of disease, 
level of human chorionic gonadotropin (hCG), duration of disease, nature of the antecedent pregnancy, and extent of prior treatment [1-4]. Based on the staging system of the International Federation of Gynecology and Obstetrics (FIGO 2002) [5], including the World Health Organization (WHO) scoring system, low-risk GTN is defined as having a FIGO/ WHO prognostic risk score lower than seven. Patients with a score of 0-6 are more likely to respond to single-agent therapy. Patients with a score $>6$ have a higher risk of resistance to single-agent chemotherapy, and are best treated with combination chemotherapy [1-4].

Most patients with low-risk GTN are cured with single-agent chemotherapy, using either methotrexate (MTX) or actinomycin-D (ActD). Several single-agent regimens have been developed with comparable remission rates [6-10]. However, non-randomized, retrospective studies [11] assessing these regimens have suggested wide variability in outcome. This variability probably results from differences in dose, frequency, route of administration and patient selection [12]. Randomized prospective trials comparing ActD with weekly MTX or 5-day MTX have shown that pulse ActD achieved significantly better response rates than weekly MTX [7], but was comparable to 5-day MTX [13]. Nonetheless, the choice of both the drug and the regimen is usually institution specific [7], and there is no consensus on the best single agent regimen.

At the New England Trophoblastic Disease Center (NETDC), an 8-day regimen alternating intramuscular MTX and folinic acid (FA) has been the preferred single-agent regimen since the early 1970s [14-16] because it is well tolerated, efficacious, and cost effective [6,16-18]. MTX infusion has also been utilized at the NETDC to both shorten and simplify the treatment [19]. While MTX has been used as primary therapy in about $90 \%$ of patients with low-risk GTN in our institution [6,20], ActD is used as first-line therapy in patients with evidence of preexisting hepatic dysfunction and as second-line therapy if there is an adverse reaction to MTX or MTX resistance.

Although the most important objective of treatment is complete remission, other considerations such as toxicity are also relevant. Chemotherapy for GTN has been associated with adverse events such as bone marrow suppression, alopecia, stomatitis/mucositis, nausea and vomiting, neuropathy, and alterations in hepatic and renal function $[7,13,21]$. However, prior analyses of MTX toxicity have been primarily restricted to cases requiring change in regimen due to adverse events [9,22-24].

Preferences in MTX dosing regimens have varied at our center over time. To appropriately evaluate effectiveness, it is important to reassess treatment outcomes and toxicity periodically. Thus, the purpose of this study was to evaluate both the outcomes and toxicity of first-line methotrexate chemotherapy in low-risk postmolar gestational trophoblastic neoplasia patients receiving 8-day methotrexate versus one-day methotrexate infusion regimens.

\section{Methods}

This retrospective study consisted of 355 patients with FIGO-defined low-risk postmolar GTN (stage I and stages II-III, score $<7$ ) [5] receiving first-line methotrexate chemotherapy who were registered in the Donald P. Goldstein, M.D., Trophoblastic Tumor Registry of the New England Trophoblastic Disease Center (NETDC) from 1974 to 2014. A final study population of 325 patients was identified after 30 patients were excluded due to using first-line therapy with weekly intramuscular MTX $(n=23$ ) or MTX $0.4 \mathrm{mg} / \mathrm{kg}$ (max. $25 \mathrm{mg}$ ) intravenously for 5 days $(n=7)$.

All electronic and paper charts for the patients were reviewed. Gestational trophoblastic neoplasia was diagnosed using FIGO 2002 criteria: an hCG level plateau of more or $<10 \%$ for at least 3 weeks, an elevation in serum hCG level $>10 \%$ over 2 weeks, or histologic evidence of choriocarcinoma. Clinical, radiologic, and laboratory assessments were carried out as described elsewhere [20,25]. Lung metastases were diagnosed by chest X-ray rather than chest CT. Magnetic resonance imaging or computed tomography scan of the brain and abdomen were performed when pulmonary involvement, liver function abnormalities, or relevant symptoms were present.

First-line therapy consisted of 8-day MTX/folinic acid [16], or oneday MTX infusion and folinic acid [19] administered as either one single course or multiple courses followed by consolidation therapy [25]. The 8-day MTX/FA regimen consisted of MTX at $1 \mathrm{mg} / \mathrm{kg}$ intramuscularly on days $1,3,5,7$ alternating with folinic acid $0.1 \mathrm{mg} / \mathrm{kg}$ orally $24 \mathrm{~h}$ after each dose of methotrexate on days $2,4,6,8$. For the one-day MTX infusion regimen, methotrexate was administered at a dose of $100 \mathrm{mg} / \mathrm{m}^{2}$ as an intravenous bolus over $30 \mathrm{~min}$ followed by a dose of $200 \mathrm{mg} / \mathrm{m}^{2}$ in a 12-hour intravenous infusion. Twenty-four hours after beginning methotrexate infusion, folinic acid was administered at a dose of $0.1 \mathrm{mg} / \mathrm{kg}$ orally every $12 \mathrm{~h}$ for four doses. If the hCG did not decline by one log and then plateaued for at least 2 consecutive weeks or re-elevated, the patient was considered resistant to MTX and switched to ActD. If the hCG did decline by 1 log or more and then plateaued or re-elevated, the patient was then again treated with the same firstline MTX regimen. Serum hCG levels were closely monitored after each course of chemotherapy. In the earlier years of this experience (1974-2009), an initial single course of methotrexate chemotherapy was administered and no further chemotherapy was given as long as the hCG level was progressively falling. If the hCG level plateaued for at least 2 consecutive weeks, re-elevated or did not decline by $>1 \log$ within 18 days of the completion of the initial treatment, the patient was considered resistant to MTX and was switched to ActD. From 2010 onwards, multiple courses of methotrexate chemotherapy were administered at fixed intervals until hCG remission, followed by 1-3 courses of consolidation chemotherapy. If the hCG level plateaued for at least 2 consecutive weeks or re-elevated, the patient was considered resistant to the first-line MTX and switched to ActD.

Chemotherapy dosing for all patients (including overweight/obese patients) was calculated based on either actual body weight or body surface area throughout the study.

Clinical data collected included age, race, BMI (weight/height ${ }^{2}$ in kg/ $\mathrm{m}^{2}$ ), history of prior mole, molar histology (complete or partial), time to persistence, pretreatment serum hCG level, use of D\&C at persistence, presence of metastatic disease, FIGO stage/risk score, first-line chemotherapy regimen (8-day MTX/FA or one-day MTX infusion) and initial treatment plan (one single course or multiple courses with consolidation), treatment outcome, and treatment-related self-reported and witnessed adverse events.

Race was self-reported as defined by the National Institutes of Health [26]. BMI was classified into 2 BMI categories [27]: overweight/ obese (BMI $\geq 25 \mathrm{~kg} / \mathrm{m}^{2}$ ) and non-overweight/obese (BMI $<25 \mathrm{~kg} / \mathrm{m}^{2}$ ). Time to persistence (days) was defined as the time between molar evacuation and postmolar GTN diagnosis. Dilatation and curettage (D\&C) at persistence was defined as occurring within 7 days after GTN diagnosis. Treatment outcome was categorized as success or failure based on the following definitions:

- Treatment success: sustained complete remission;

- Treatment failure: need to switch regimens due to resistance, relapse, substantial toxicity;

- Sustained complete remission: normalization of serum hCG levels for 3 consecutive weeks and then monthly for 1 year;

- Resistance: hCG plateau of $\pm 10 \%$ over the course of 2 weeks or a reelevation in at least one measurement of hCG level;

- Relapse: hCG re-elevation after three normal weekly tests, in the absence of a new pregnancy;

- Substantial toxicity: treatment-related grade 3-4 adverse event requiring need to switch to second-line chemotherapy.

Adverse events were defined and classified according to standardized criteria (Common Terminology Criteria for Adverse Events-CTCAE v4.0). Complete blood cell count, platelet count, and renal (serum 
creatinine) and liver function tests (aspartate aminotransferase, blood bilirubin) were regularly assessed during chemotherapeutic treatment.

Statistical analyses were carried out using SPSS statistical software (version 21.0, SPSS, Inc., Chicago, IL). Patient characteristics and treatment outcome across type of MTX regimens (8-day MTX/FA vs oneday MTX infusion) were compared using the Mann-Whitney test (for non-parametric testing), Chi-square test (for comparison across groups), and Fisher exact test (for proportions) as appropriate. Additionally, treatment outcome by type of MTX regimen was assessed using multiple logistic regression adjusted for age and race. The association of MTX regimens with adverse events was analyzed by the Chisquare and Fisher exact tests as indicated. For all analyses, an alpha level $<0.05$ was considered statistically significant.

This study was approved by the Institutional Review Board (\# 2004P001372/BWH) at Brigham and Women's Hospital.

\section{Results}

The final study population consisted of 325 patients. Adverse event analysis was possible for 308 patients, due to missing data on 17 patients. All patients were followed with weekly hCG levels until undetectable for 3 consecutive weeks and then monthly hCG levels until undetectable for 12 consecutive months.

Patient and disease characteristics by type of methotrexate regimen are shown in Table 1 . There were significant differences between the groups regarding patient age, race, and BMI $\geq 25 \mathrm{~kg} / \mathrm{m}^{2}$, but no significant differences in disease presentation, extent of disease, or initial treatment plan $(p=0.417)$.

A temporal relationship was noted according to dosing regimen. In the time period 1974-1984, 8-day MTX/FA was the preferred first-line treatment. Between 1985 and 2009, the use of one-day MTX infusion was more frequent, and from 2010 onward 8-day MTX/FA has been once again the preferred treatment (Fig. 1).
Significant differences were observed in sustained remission rates between the 8-day MTX/FA (84\%) and one-day MTX infusion (62\%) ( $p$ $<0.001$ ) (Table 2). Multiple logistic regression analysis adjusted for age and race revealed a three-fold greater chance for sustained remission using the 8-day MTX/FA regimen compared to the one-day MTX infusion $(\mathrm{OR}=3.08,95 \% \mathrm{CI}=1.76-5.37, p<0.001)$.

The overall failure rates are further understood by comparing methotrexate resistance, relapse, and toxicity requiring a change in chemotherapy between the two regimens. Methotrexate-resistance accounted for treatment failure in $7.3 \%$ of the patients treated with 8 day-MTX/FA and in $34.5 \%$ of those given one-day MTX infusion ( $p<$ 0.001 ). The relapse rate (3\%) was similar between MTX regimens. Relapse occurred in 11 of 288 (3.8\%) patients treated with planned 1 course of MTX versus none of the 37 patients treated with repetitive courses of MTX and consolidation $(p=1.0)$ (Table S1). The mean time for relapse was 3 months (range of 1 to 8 months), with $82 \%$ of the relapses occurring within the first six months after remission. Treatment-related grade 3-4 adverse events requiring switching to second-line chemotherapy occurred in none of the patients receiving one-day MTX infusion, and in 8 (5.3\%) of those treated with 8-day MTX/FA ( 6 cases of grade 3 elevated liver function test results, 2 cases of grade 3 oral mucositis) ( $p=0.001$ ) (Table 2 ).

Asian patients have been previously reported to show more resistance to first-line MTX chemotherapy [23]. As only two Asian patients received the 8-day MTX/FA regimen, this could distort a comparison of the regimens. However, among non-Asian patients, methotrexateresistance still occurred in $6.4 \%$ of patients treated with 8-day MTX/FA and $34.4 \%$ of those given one-day MTX infusion $(p<0.001$, Table 3$)$.

Out of 90 patients (27.7\%) who experienced failure of first-line MTX chemotherapy, 68 patients (75.5\%) were treated with ActD and the success rate of ActD as second-line treatment was 80.8\% (55/68 patients). Overall, 35 patients who experienced failure of first-line MTX chemotherapy required multiagent chemotherapy (either as second-line treatment or for actinomycin D-failure) and/or surgery to be cured.

Table 1

Patient and disease characteristics by type of methotrexate regimen.

\begin{tabular}{|c|c|c|c|c|}
\hline Characteristic & $\begin{array}{l}\text { Overall } \\
(n=325)\end{array}$ & $\begin{array}{l}\text { 8-day MTX/FA } \\
(n=151)\end{array}$ & $\begin{array}{l}\text { One-day MTX infusion } \\
(n=174)\end{array}$ & $p$ \\
\hline Age $\left(\right.$ years) ${ }^{a}$ & $28(14-50)$ & $27(14-49)$ & $30(15-50)$ & $<0.001^{(1)}$ \\
\hline \multicolumn{5}{|l|}{ Race (\%) } \\
\hline White & 85.9 & 92.4 & 80.5 & \multirow[t]{4}{*}{$0.002^{(3)}$} \\
\hline African American & 5.6 & 4.8 & 6.3 & \\
\hline Asian & 6.3 & 1.4 & 10.3 & \\
\hline Unknown & 2.2 & 1.4 & 2.9 & \\
\hline $\mathrm{BMI} \geq 25 \mathrm{~kg} / \mathrm{m}^{2}(\%)^{\mathrm{b}}$ & 25.8 & 19.8 & 30.1 & $0.046^{(2)}$ \\
\hline Prior mole $(\%)^{\mathrm{c}}$ & 3.1 & 1.3 & 4.7 & $0.112^{(3)}$ \\
\hline \multicolumn{5}{|l|}{ Molar histology (\%) } \\
\hline Partial & 16.3 & 9.9 & 21.8 & \multirow[t]{2}{*}{$0.004^{(2)}$} \\
\hline Complete & 83.7 & 90.1 & 78.2 & \\
\hline Time to persistence (days) ${ }^{\mathrm{a}}$ & $47(7-210)$ & $47(12-210)$ & $47(7-185)$ & $0.731^{(1)}$ \\
\hline Pretreatment hCG $(\mathrm{mIU} / \mathrm{mL})^{\mathrm{a}}$ & $1410(7-193,000)$ & $1600(15-126,197)$ & $1207(7-193,000)$ & $0.486^{(1)}$ \\
\hline $\mathrm{D} \& \mathrm{C}(\%)$ & 70.7 & 68 & 73 & $0.325^{(2)}$ \\
\hline Metastatic disease (\%) & 10.5 & 9.9 & 10.9 & $0.772^{(2)}$ \\
\hline \multicolumn{5}{|l|}{ Stage (\%) } \\
\hline I & 89.5 & 90.1 & 89.1 & \multirow[t]{3}{*}{$1.000^{(3)}$} \\
\hline II & 0.9 & 0.7 & 1.1 & \\
\hline III & 9.5 & 9.3 & 9.8 & \\
\hline FIGO score ${ }^{\mathrm{a}}$ & $1(0-6)$ & $1(0-6)$ & $1(0-5)$ & $0.241^{(1)}$ \\
\hline FIGO score $>4(\%)$ & 2.8 & 2.6 & 2.9 & $1.000^{(3)}$ \\
\hline \multicolumn{5}{|l|}{ Initial treatment plan (\%) } \\
\hline Single course & 89 & 87 & 90 & \multirow[t]{2}{*}{$0.417^{(2)}$} \\
\hline Multiple courses & 11 & 13 & 10 & \\
\hline
\end{tabular}

a Data are reported as medians. The numbers in parentheses represent range.

b Information not available in 25 patients.

c Information not available in 3 patients.

1 Mann-Whitney.

2 Chi-square test.

${ }^{3}$ Fisher exact test. 


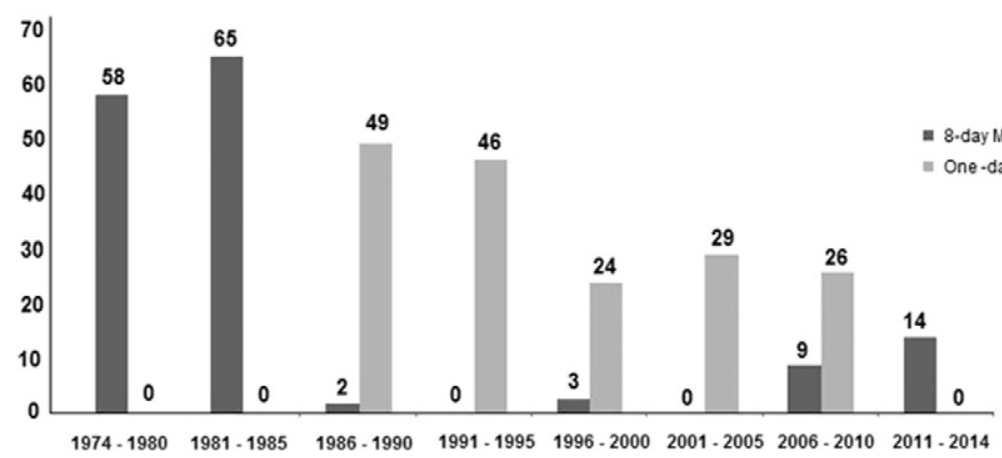

Fig. 1. Choice of methotrexate regimen over the years.

Three patients underwent surgery: 1 hysterectomy, 1 local uterine resection, and 1 lung resection. All 90 patients who experienced failure of first-line MTX chemotherapy achieved sustained complete remission with these treatments.

With planned 1 course of MTX, the median number of courses of 8day MTX/FA and one-day MTX infusion to disease remission or change in medication was 1 (range 1-6) and 1 (range 1-3), respectively ( $p$ $=1.0$ ). With planned multiple courses of MTX, the median number of courses of 8-day MTX/FA and one-day MTX infusion to disease remission or change in medication was 2 (range 1-6) and 2 (range 1-4), respectively $(p=1.0)$ (Table S1).

With planned 1 course of MTX, the median time to remission or change in medication with 8-day MTX/FA and one-day MTX infusion was 40 days (range 10-376) versus 45.5 days (range 12-296), respectively $(p<0.0001)$. With planned multiple courses of MTX, the median time to remission or change in medication with 8-day MTX/FA and oneday MTX infusion was 44.5 days (range 29-126) and 73 (21-132), respectively $(p=1.0)$ (Table S1).

System organ class adverse events (SOC) were more frequent using 8-day MTX/FA (83.3\%) than using one-day MTX infusion (57.6\%, $p<$ 0.001 ) (Table 4). Gastrointestinal disorders and abnormal laboratory findings (SOC investigations), followed by eye disorders, general disorders and infections were the most frequent adverse events with both regimens. However, with both MTX regimens, most patients experienced only mild (grade 1 or 2) chemotherapy-related toxicity (Table S2).

Compared to one-day MTX infusion, 8-day MTX/FA was associated with significantly higher rates of gastrointestinal disorders (48\% vs $24 \%$ ), abnormal laboratory findings ( $48 \%$ vs $28 \%$ ), eye disorders ( $37 \%$ vs $19 \%$ ) and general disorders ( $22 \%$ vs $5 \%)(p<0.001)$. Only the frequency of infections did not significantly differ between 8-day MTX/FA and one-day MTX infusion ( $20 \%$ vs $12 \%, p=0.083$ ) (Table 4 ).

The most common gastrointestinal adverse events were oral mucositis, nausea and abdominal pain, with patients receiving 8-day MTX/FA showing a significantly higher rate of oral mucositis compared to those

Table 2

Treatment outcome by type of methotrexate regimen.

\begin{tabular}{lllll}
\hline Outcome & $\begin{array}{l}\text { Overall } \\
(n=325)\end{array}$ & $\begin{array}{l}\text { 8-day MTX/FA } \\
(n=151)\end{array}$ & $\begin{array}{l}\text { One-day MTX } \\
\text { infusion } \\
(n=174)\end{array}$ & $p$ \\
& & & & \\
\hline $\begin{array}{l}\text { Success } \\
\text { Sustained remission (\%) }\end{array}$ & 72.3 & 84.1 & 62.1 & $<0.001^{\text {a }}$ \\
$\begin{array}{l}\text { Failure } \\
\quad \text { Resistance (\%) }\end{array}$ & 21.8 & 7.3 & 34.5 & $<0.001^{\text {a }}$ \\
$\quad \begin{array}{l}\text { Relapse (\%) } \\
\text { Substantial toxicity (\%) }\end{array}$ & 2.4 & 3.3 & 3.4 & $0.810^{\mathrm{a}}$ \\
\end{tabular}

Crude odds ratio $=3.23,95 \% \mathrm{CI}=1.90-3.51, p$-value $<0.001$.

Adjusted* odds ratio $=3.08,95 \% \mathrm{CI}=1.76-5.37, p$-value $<0.001$.

*For age and race.

a Chi-square test.

b Fisher exact test. receiving one-day MTX infusion ( $40 \%$ vs $10 \%, p<0.001$ ). The abnormal laboratory findings most frequently observed were decreased neutrophil count (neutropenia), anemia (blood system disorders) and increased aspartate aminotransferase (SGOT). The frequency of each of these adverse events was significantly higher in patients treated with 8-day MTX/FA (Table 4).

Conjunctivitis and dry eye were the most common eye disorders, with no significant difference between MTX regimens. The adverse events within SOC general disorders most frequently found were fatigue and fever with significantly higher rates with 8-day-MTX/FA. Fever was associated with infection in $92 \%$ of the cases. The infections most commonly seen were pharyngitis, vaginal infection (vulvovaginitis), upper respiratory infection, urinary tract infection and uterine infection (endometritis). However, only pharyngitis $(p=0.002)$ and uterine infection $(p=0.039)$ rates were significantly higher in the 8-day MTX-FA regimen (Table 4 continuation). In patients who developed methotrexate-induced neutropenia, the rate of infectious events was higher (8-day MTXFA $=30 \%$, one-day MTX infusion = 14\%) compared to those without neutropenia (8-day MTXFA $=16 \%$, one-day MTX infusion $=12 \%$, although these differences were not significant (Table S3).

Hospitalization or prolongation of existing hospitalization due to treatment-related toxicity occurred in $3.2 \%$ (10/308) of the cases, all of them treated with 8-day MTX/FA (6.6\%, 10/151). These cases included oral mucositis ( 4 patients), infections (4 patients), pleuritic pain ( 2 patients) and abdominal pain/peritonitis (1 patient) (Table S4).

The median number of adverse events following 1 course of MTX versus multiple courses of MTX was 1 (range $0-9$ ) versus 2 (range $0-9)(p=0.002)$.

\section{Discussion}

Methotrexate remains the most commonly used drug for treatment of low-risk GTN, but there have been few large, detailed studies looking at the clinical tradeoffs and toxicities of different dosing regimens. At

Table 3

Treatment outcome by type of methotrexate regimen in non-Asian patients (White and African American).

\begin{tabular}{lllll}
\hline Outcome & $\begin{array}{l}\text { Overall } \\
(n=292)\end{array}$ & $\begin{array}{l}\text { 8-day MTX/FA } \\
(n=141)\end{array}$ & $\begin{array}{l}\text { One-day MTX } \\
\text { infusion } \\
(n=151)\end{array}$ & $p$ \\
\hline $\begin{array}{llll}\text { Sustained remission (\%) } \\
\text { Failure }\end{array}$ & $212(72.6 \%)$ & $119(84.4 \%)$ & $93(61.6 \%)$ & $<0.001^{\text {a }}$ \\
$\quad$ Resistance (\%) & $61(20.8 \%)$ & $9(6.4 \%)$ & $52(34.4 \%)$ & $<0.001^{\text {a }}$ \\
$\quad \begin{array}{l}\text { Relapse (\%) } \\
\text { Substantial toxicity (\%) }\end{array}$ & $8(2.8 \%)$ & $8(3.6 \%)$ & $6(4.0 \%)$ & $0.907^{\mathrm{a}}$ \\
\hline
\end{tabular}

Crude odds ratio $=3.37,95 \%$ IC $=1.92-5.91 ; p$-value $<0.001$.

Adjusted* odds ratio $=3.16,95 \% \mathrm{IC}=1.79-5.59 ;$-value $<0.001$.

*For age.

a Chi-square test.

b Fisher exact test. 
Methotrexate related adverse events by type of methotrexate regimen.

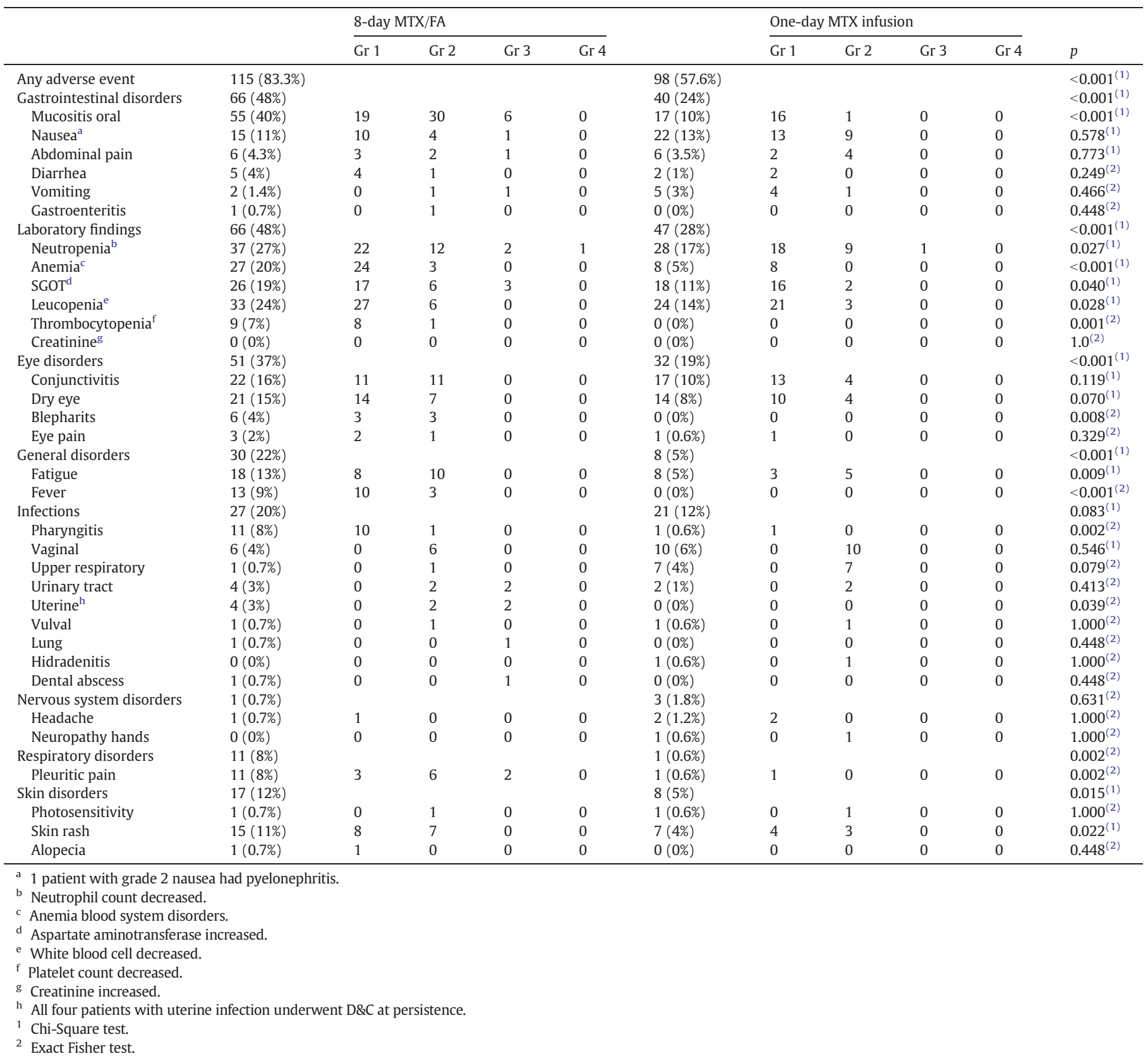

NETDC, the success of primary treatment with 8-day MTX/FA was 3-fold higher than with one-day MTX infusion. While treatment-related adverse events were more frequent with 8-day MTX/FA regimen (83\%) than with one-day MTX infusion (57\%), most patients experienced only mild (grade 1 or 2 ) chemotherapy-related toxicity with both MTX regimens. While grade 1 and 2 toxicities did not necessitate a change in treatment, it can meaningfully impact the quality of life of patients.

Notably, there were no differences in disease presentation, extent of disease, and initial treatment plan between the two regimens. However, patient age, race, and BMI $\geq 25 \mathrm{~kg} / \mathrm{m}^{2}$ significantly differed. Given that BMI has been previously demonstrated to have no influence on the response to chemotherapy in low-risk GTN [28], the present analysis of treatment outcomes was adjusted only for age and race.

Our results show that the choice of regimen changed in the NETDC over the years. One-day MTX infusion was more frequently utilized between 1985 and 2009 with the hope it would lead to a high remission rate and be less burdensome and disruptive to patients than 8-day MTX/FA. As it became increasingly apparent that one-day MTX infusion was less effective than the 8-day MTX/FA regimen, its use was discontinued and the 8-day regimen was resumed in 2010.

Sustained remission rate among our patients significantly differed between regimens, with a three-fold greater chance for sustained remission using 8-day MTX/FA. Similar rates have been reported in previous studies $[16,18,19]$. The lower remission rate achieved with one-day MTX infusion may be attributable to the duration of cell exposure to chemotherapy. Cell survival is affected more by exposure duration than by exposure dose [29]. With one-day infusion regimens, the blood and tissue levels may not be sustained at cytotoxic levels for sufficient time to kill all multiplying trophoblastic cells. The 8-day MTX/FA regimen, in turn, affords increased exposure to the drug [30]. Methotrexate-resistance accounted for treatment failure significantly more frequently in our patients given one-day MTX infusion than in those receiving 8-day-MTX/FA, despite the fact that resistance 
prognostic factors for failure (pretreatment hCG levels, metastatic disease, FIGO score $>4$ ) were similar in both groups.

With both 8-day MTX/FA and one-day MTX infusion, the relapse rate was low (3\%), even though the large proportion of patients (90\%) were treated with one single course without consolidation. This rate of relapse is similar to those reported in other studies that ranged between $2 \%$ and $4 \%[22,31-35]$. Similarly, the timing of relapse in our patients was consistent with that reported in the literature [22,31-35]. However, because other studies used different definitions of relapse, a direct comparison of results is not possible.

Switching to second-line chemotherapy due to treatment-related grade 3-4 adverse events was observed in $5.3 \%$ of the patients receiving 8-day MTX/FA, a rate similar to those found in other centers using this regimen [21,34]. With one-day MTX infusion, no patient required change to a second-line regimen due to treatment-related grade 3-4 adverse events. This confirmed previous results observed in a prior study conducted in our center [19].

Overall, system organ class adverse events (SOC) were more frequent with 8-day MTX/FA (83.3\%) than with one-day MTX infusion (57.6\%). The frequency rate of SOC adverse events found with one-day MTX infusion was comparable to those previously reported [19]. However, with 8-day MTX/FA, toxicity was higher than reported in the literature [21]. This may be due partially to the fact that as part of our safety and quality measures, our patients are not only encouraged to call whenever they have any questions or concerns regarding their treatment, but are also contacted weekly about treatment adverse effects. Nonetheless, most of our patients experienced only mild (grade 1 or 2) chemotherapy-related toxicity with both regimens, as observed by others [21,34].

Compared to one-day MTX infusion, 8-day MTX/FA was associated with significantly higher rates of gastrointestinal disorders, abnormal laboratory findings, eye disorders and general disorders, as well as hospitalization. The frequency of infections did not significantly differ between regimens.

Gastrointestinal disorders have been reported as the most common side effects that can affect up to 70\% of patients receiving MTX [36]. The most common gastrointestinal adverse event observed in our study was oral mucositis at a rate significantly higher with 8-day MTX/FA compared to one-day MTX infusion (40\% vs $10 \%$ ). Although almost all chemotherapy drugs have a potential to cause mucositis, agents that affect DNA synthesis and are S-phase specific, such as MTX, are expected to cause the most mucositis [37]. Additionally, MTX is secreted in the saliva thus increasing oral toxicity. Chemotherapy-induced mucositis damages the submucosal components early after chemotherapy, and sets up a cascade of events that ultimately lead to epithelial lesions that manifest 7-10 days later. Mucositis then gradually subsides, leaving no scars, over a period of 2-3 weeks after drug administration [38]. Mucositis is also a major cause of hospitalization or switching to other drugs [21,22,38].

The most commonly observed abnormal laboratory findings among our patients were decreased neutrophil count (neutropenia), anemia (blood system disorders) and hepatoxicity, which were significantly more frequent with 8-day MTX/FA. Conjunctivitis and dry eye were the most common eye disorders, with no significant difference between MTX regimens. Indeed, MTX alters the normal tear film physiology either by causing inflammation of the lacrimal glands or by being excreted directly into tears. The antimetabolic nature of methotrexate is most effective against rapidly dividing cells; therefore, the hematological, gastrointestinal, and ocular systems, which feature a high turnover of cells are the most likely to display signs and symptoms of toxicity. These side effects have been greatly ameliorated using folinic acid (a form of tetrahydrofolate) $[39,40]$, which is used to protect against MTX toxicity in normal cells by overcoming the defect in folate metabolism induced by MTX.

The adverse events within SOC general disorders most frequently found were fatigue and fever with significantly higher rates among patients receiving 8-day-MTX/FA. Fatigue has been reported in $80 \%$ to $99 \%$ of patients who undergo anti-cancer treatment. The importance of physical, psychological, and social factors is usually unclear, but these factors appear to be important in the pathogenesis of fatigue and may be predominate in some cases [41]. Fever was associated with infection in $92 \%$ of our patients. Low doses of MTX usually do not increase the probability of opportunistic infections [36]. However, infectious events (with focus identification) were seen in $20 \%$ and $12 \%$ of our patients receiving 8-day MTX and one-day MTX infusion, respectively. MTX therapy can suppress the immune system and make patients more susceptible to infection as therapy directly affects the production of neutrophils and produces antiproliferative effects on lymphocytes [42].

Comparing our findings with those previously reported is difficult because studies specifically focusing on MTX adverse effects in patients with GTN are mainly limited to toxicity resulting in the need to change treatment. Additionally, underreporting, differences in terminology and different assessment techniques hinder the actual understanding of the severity and frequency of toxicity in clinical practice.

Our results show that while being more toxic than one-day MTX infusion, 8-day MTX/FA was associated with a higher remission rate. The success of the first regimen is of particular importance because it limits the exposure of most patients to combination chemotherapy, and reduces the social costs of the treatment. Moreover, primary treatment failure is a major source of psychological distress for patients with GTN [43], who are usually women of reproductive and working age. It results in an increase in the overall treatment duration, and delays the ability of women to conceive as well as return to work. Given this and its higher effectiveness, 8-day MTX/FA remains the treatment of choice at the NETDC for patients with low-risk postmolar GTN.

In conclusion, this is one of the largest studies to comprehensively catalogue toxicities associated with 8-day MTX/FA and one-day MTX infusion in low-risk postmolar GTN. Although treatment-related adverse events were more frequent with the 8-day MTX/FA regimen these were all self-limited, primarily grade 1 and 2, and resolved with no long-term consequences. Because underreporting of toxicities is common and often restricted to severe toxicity, this study can be used for comparison with future reports on the broad range of MTX treatmentrelated toxicity.

\section{Conflict of interest}

The authors declare no conflict of interest.

\section{Acknowledgements}

The authors are thankful for the support of the São Paulo Research Foundation-FAPESP (grant \#2015/12530-0), the Donald P. Goldstein, MD Trophoblastic Tumor Registry Endowment, and the Dyett Family Trophoblastic Disease Research and Registry Endowment.

\section{Appendix A. Supplementary data}

Supplementary data to this article can be found online at https://doi. org/10.1016/j.ygyno.2017.10.028.

\section{References}

[1] H.Y. Ngan, H. Bender, J.L. Benedet, H. Jones, G.C. Montruccoli, S. Pecorelli, et al., Gestational trophoblastic neoplasia, FIGO 2000 staging and classification, Int. J. Gynecol. Obstet. 83 (Suppl. 1) (2003) 175-177.

[2] M.J. Seckl, N.J. Sebire, R.S. Berkowitz, Gestational trophoblastic disease, Lancet 376 (2010) 717-729

[3] J.R. Lurain, Gestational trophoblastic disease II: classification and management of gestational trophoblastic neoplasia, Am. J. Obstet. Gynecol. 204 (2011) 11-18.

[4] J. Brown, R.W. Naumann, M.J. Seckl, J. Schink, 15 years of progress in gestational trophoblastic disease: scoring, standardization, and salvage, Gynecol. Oncol. 144 (2017) 200-207. 
[5] FIGO Oncology Committee, FIGO staging for gestational trophoblastic neoplasia 2000, Int. J. Gynecol. Obstet. 77 (2002) 285-287.

[6] W.B. Growdon, A.J. Wolfberg, D.P. Goldstein, C.M. Feltmate, M.E. Chinchilla, E.S Lieberman, et al., Evaluating methotrexate treatment in patients with low-risk postmolar gestational trophoblastic neoplasia, Gynecol. Oncol. 112 (2009) 353-357.

[7] R.J. Osborne, V. Filiaci, J.C. Schink, R.S. Mannel, A. Alvarez Secord, J.L. Kelley, et al., Phase III trial of weekly methotrexate or pulsed dactinomycin for low-risk gestational trophoblastic neoplasia: a Gynecologic Oncology Group Study, J. Clin. Oncol. 29 (2011) 825-831.

[8] D. Sita-Lumsden, I. Short, N.J. Lindsay, D. Sebire, M.J. Seckl Adjogatse, et al., Treatment outcomes for 618 women with gestational trophoblastic tumours following a molar pregnancy at the Charing Cross Hospital, 2000-2009, Br. J. Cancer 107 (2012) 1810-1814.

[9] E. Chapman-Davis, A.V. Hoekstra, A.W. Rademaker, J.C. Schink, J.R. Lurain, Treatment of nonmetastatic and metastatic low-risk gestational trophoblastic neoplasia: factors associated with resistance to single-agent methotrexate chemotherapy, Gynecol. Oncol. 125 (2012) 572-575.

[10] F. Taylor, T. Grew, J. Everard, L. Ellis, M.C. Winter, J. Tidy, et al., The outcome of patients with low risk gestational trophoblastic neoplasia treated with single agent intramuscular methotrexate and oral folinic acid, Eur. J. Cancer 49 (2013) 3184-3190.

[11] T.A. Lawrie, M. Alazzam, J. Tidy, B.W. Hancock, R. Osborne, First-line chemotherapy in low-risk gestational trophoblastic neoplasia, Cochrane Database Syst. Rev. 6 (2016), CD007102. https://doi.org/10.1002/14651858.CD007102.pub4 (Review).

[12] M.J. Seckl, N.J. Sebire, R.A. Fisher, F. Golfier, L. Massuger, C. Sessa, et al., Gestationa trophoblastic disease: ESMO Clinical Practice Guidelines for diagnosis, treatment and follow-up, Ann. Oncol. 24 (Suppl. 6) (2013) vi39-50, https://doi.org/10.1093/ annonc/mdt345.

[13] F. Yarandi, A. Mousavi, F. Abbaslu, S. Aminimoghaddam, S. Nekuie, K. Adabi, et al., Five-day intravascular methotrexate versus biweekly actinomycin-D in the treatment of low-risk gestational trophoblastic neoplasia: aclinical randomized trial, Int. J. Gynecol. Cancer 26 (2016) 971-976.

[14] R.S. Berkowitz, D.P. Goldstein, Methotrexate with citrovorum factor rescue for nonmetastatic gestational trophoblastic neoplasms, Obstet. Gynecol. 54 (1979) 725-728.

[15] R.S. Berkowitz, D.P. Goldstein, M.R. Bernstein, Methotrexate with citrovorum factor rescue as primary therapy for gestational trophoblastic disease, Cancer 50 (1982) 2024-2027.

[16] R.S. Berkowitz, D.P. Goldstein, M.R. Bernstein, Ten year's experience with methotrexate and folinic acid as primary therapy for gestational trophoblastic disease, Gynecol. Oncol. 23 (1986) 111-118.

[17] N.T. Shah, L. Barroilhet, R.S. Berkowitz, D.P. Goldstein, N. Horowitz, A cost analysis of first-line chemotherapy for low-risk gestational trophoblastic neoplasia, J. Reprod. Med. 57 (2012) 211-218

[18] D.P. Goldstein, R.S. Berkowitz, N.S. Horowitz, Optimal management of low-risk gestational trophoblastic neoplasia, Expert. Rev. Anticancer. Ther. 15 (2015) 1293-1304.

[19] A.P. Garrett, E.O. Garner, D.P. Goldstein, R.S. Berkowitz, Methotrexate infusion and folinic acid as primary therapy for nonmetastatic and low-risk metastatic gestational trophoblastic tumors. 15 years of experience, J. Reprod. Med. 47 (2002) 355-362.

[20] I. Maestá, W.B. Growdon, D.P. Goldstein, M.R. Bernstein, N.S. Horowitz, M.V. Rudge et al., Prognostic factors associated with time to hCG remission in patients with lowrisk postmolar gestational trophoblastic neoplasia, Gynecol. Oncol. 130 (2013) 312-316.

[21] F. Khan, J. Everard, S. Ahmed, R.E. Coleman, M. Aitken, B.W. Hancock, Low-risk persistent gestational trophoblastic disease treated with low-dose methotrexate: efficacy, acute and long-term effects, Br. J. Cancer 89 (2003) 2197-2201.

[22] I.A. McNeish, S. Strickland, L. Holden, G.J. Rustin, M. Foskett, MJ. Seckl, et al, Lowrisk persistent gestational trophoblastic disease: outcome after initial treatment with low-dose methotrexate and folinic acid from 1992 to 2000, J. Clin. Oncol. 20 (2002) 1838-1844

[23] I. Maestá, R.S. Berkowitz, D.P. Goldstein, M.R. Bernstein, L.A. Ramírez, N.S. Horowitz Relationship between race and clinical characteristics, extent of disease, and response to chemotherapy in patients with low-risk gestational trophoblastic neoplasia, Gynecol. Oncol. 138 (2015) 50-54.

[24] M.C. Winter, J.A. Tidy, A. Hills, J. Ireson, S. Gillett, K. Singh, et al., Risk adapted singleagent dactinomycin or carboplatin for second-line treatment of methotrexate resistant low-risk gestational trophoblastic neoplasia, Gynecol. Oncol. 143 (2016) 565-570.

[25] D.P. Goldstein, R.S. Berkowitz, Current management of gestational trophoblastic neoplasia, Hematol. Oncol. Clin. North Am. 26 (2012) 111-131.

[26] National Institutes of Health, NIH Policy on Reporting Race and Ethnicity Data: Subjects in Clinical Research, NIH, Bethesda, 2001 http:/grants.nih/gov/grants/guide/ noticefiles/NOT-OD-01-053.html (accessed 15.06.17).

[27] World Health Organization, Obesity and Overweight Fact Sheet 311, WHO, Geneva, 2016 http://www.who.int/mediacentre/factsheets/fs311/en/ (accessed 15.06.17).

[28] I. Maestá, N.S. Horowitz, D.P. Goldstein, M.R. Bernstein, L.A. Ramírez, J. Moulder, et al., Response to chemotherapy in overweight/obese patients with low-risk gestational trophoblastic neoplasia, Int. J. Gynecol. Cancer 25 (2015) 734-740.

[29] H. Eichholtz, K.R. Trott, Effect of methotrexate concentration and exposure time on mammalian cell survival in vitro, Br. J. Cancer 41 (1980) 277-284.

[30] E.I. Kohorn, Is lack of response to single-agent chemotherapy in gestational trophoblastic disease associated with dose scheduling or chemotherapy resistance? Gynecol. Oncol. 85 (2002) 36-39.

[31] H. Matsui, K. Suzuka, K. Yamazawa, N. Tanaka, A. Mitsuhashi, K. Seki, et al., Relapse rate of patients with low-risk gestational trophoblastic tumor initially treated with single-agent chemotherapy, Gynecol. Oncol. 96 (2005) 616-620.

[32] K.K. Chan, Y. Huang, K.F. Tam, K.Y. Tse, H.Y. Ngan, Single-dose methotrexate regimen in the treatment of low-risk gestational trophoblastic neoplasia, Am. J. Obstet. Gynecol. 195 (2006) 1282-1286.

[33] T. Powles, P.M. Savage, J. Stebbing, D. Short, A. Young, M. Bower, et al., A comparison of patients with relapsed and chemo-refractory gestational trophoblastic neoplasia, Br. J. Cancer 96 (2007) 732-737.

[34] G.E. Chalouhi, F. Golfier, P. Soignon, J. Massardier, J.P. Guastalla, V. Trillet-Lenoir, et al., Methotrexate for 2000 FIGO low-risk gestational trophoblastic neoplasia patients: efficacy and toxicity, Am. J. Obstet. Gynecol. 200 (2009) (643.e1-6).

[35] C. Lybol, F.C. Sweep, R. Harvey, H. Mitchell, D. Short, C.M. Thomas, et al., Relapse rates after two versus three consolidation courses of methotrexate in the treatment of low-risk gestational trophoblastic neoplasia, Gynecol. Oncol. 125 (2012) 576-579.

[36] M.D. Schafranski, A.B. Merlini, E.A.C. Araújo, N.L.B. Camargo, P. Arruda, Methotrexate: Update on Pharmaclology, Clinical Applications and Warmings, Nova Science Publishers, New York, 2012 https://www.novapublishers.com/catalog/product info.php?products_id =34660\&osCsid = 5e9a553a1b1129fed10cc0ad3598a587 (accessed 15.06.17).

[37] M.U. Naidu, G.V. Ramana, P.U. Rani, I.K. Mohan, A. Suman, P. Roy, Chemotherapyinduced and/or radiation therapy-induced oral mucositis - complicating the treatment of cancer, Neoplasia 6 (2004) 423-431.

[38] B. Chaveli-López, J.V. Bagán-Sebastián, Treatment of oral mucositis due to chemotherapy, J. Clin. Exp. Dent. 8 (2016) e201-9.

[39] K.D. Bagshawe, J. Dent, E.S. Newlands, R.H. Begent, G.J. Rustin, The role of low-dose methotrexate and folinic acid in gestational trophoblastic tumours (GTT), Br. J. Obstet. Gynaecol. 96 (1989) 795-802.

[40] M.M. Skubisz, S. Tong, The evolution of methotrexate as a treatment for ectopic pregnancy and gestational trophoblastic neoplasia: a review, ISRN Obstet. Gynecol. 2012 (2012) 637094, https://doi.org/10.5402/2012/637094.

[41] G.A. Curt, W. Breitbart, D. Cella, J.E. Groopman, S.J. Horning, L.M. Itri, et al., Impact of cancer-related fatigue on the lives of patients: new findings from the fatigue coalition, Oncologist 5 (2000) 353-360.

[42] M. Cutolo, A. Sulli, C. Pizzorni, B. Seriolo, R.H. Straub, Anti-inflammatory mechanisms of methotrexate in rheumatoid arthritis, Ann. Rheum. Dis. 60 (2001) 729-735.

[43] L. Wenzel, R.S. Berkowitz, E. Newlands, B. Hancock, D.P. Goldstein, M.J. Seckl, et al., Quality of life after gestational trophoblastic disease, J. Reprod. Med. 47 (2002) 387-394. 